\title{
Torsion of a Wandering Spleen
}

\author{
T HAQUE $^{\mathrm{a}}$, S HOSSAIN ${ }^{\mathrm{b}}$, KABMT ALAM ${ }^{\mathrm{c}}$, MA RAHMAN ${ }^{\mathrm{d}}$
}

\begin{abstract}
Summary:
Wandering spleen is a rare clinical condition characterized by ectopic positioning of the spleen due to abnormal peritoneal attachments including the lienorenal and gastrosplenic ligaments. The spleen can "wander" or migrate into various positions within the abdomen or pelvis due to this ligamentous laxity. The clinical presentation of patients with this entity is variable and can range from an incidental finding to an acute abdomen associated with torsion. Various imaging modalities can be utilized for the diagnosis of this condition.A variable treatment options
\end{abstract}

\section{Introduction:}

Wandering spleen is a rare clinical entity. It usually occurs at $20-40$ years of age and most cases are seen in women and is rare in children. ${ }^{1}$

Torsion of the wandering spleen is a rare cause of acute abdominal pain. A “wandering spleen” is characterized by laxity or absence of the supporting splenic ligaments and where a long pedicle facilitates abnormal positioning of the spleen outside its native left sub-diaphragmatic location. ${ }^{2}$ Torsion may vary from $1 / 2$ to 6 complete turns around its axis depending upon the weight of spleen, length of pedicle and degree of ligamentous laxity. ${ }^{3}$

Majority of the cases of wandering spleen present as an acute abdomen due to splenic torsion and subsequent splenic infarction. ${ }^{4}$

Clinical presentation of wandering spleen is variable. Acute torsion may precipitate fever, vomiting and acute abdominal pain, while recurrent chronic torsion and detorsion may presents as intermittent colicky abdominal pain or vague abdominal discomfort. ${ }^{6}$

a. Dr. Taufiqul Haque. FCPS (Surgery), MRCS (Ire, UK) Assistant Professor Dept. of Surgery, Shaheed Monsur Ali Medical College, Uttara, Dhaka

b. Dr. Shamim Hossain FCPS, MRCS, Assistant Professor, Dept. of Surgery Shaheed Monsur Ali Medical College. Uttara Dhaka.

c. Dr. K.A.B.M Taiful Alam. FCPS, Associate Professor, Dept. of Surgery Shaheed Monsur Ali Medical College. Uttara, Dhaka.

d. Prof. Md. Atiqur Rahman. FCPS (Surgery), FWHO(Urology) Professor of Surgery, Shaheed Monsur Ali Medical College Uttara , Dhaka.

Address of Correspondence: Dr. Taufiqul Haque, Assistant Professor, Department of Surgery, Shaheed Monsur Ali Medical College and Hospital. Uttara, Dhaka.

Received: 27 December, 2012

Accepted: 10 June, 2013 for torsion of wandering spleen include splenectomy or splenopexy.

Here we described a case of 32 years young lady with acute abdomen due to torsion of a wandering spleen. Diagnosis was made on the basis of clinical findings and abdominal ultrasound. Laparotomy was performed and the infarcted spleen was removed.

Keywords: Splenic torsion, splenectomy, wandering spleen.

(J Banagladesh Coll Phys Surg 2013; 31: 219-222)

The non-specific signs and symptoms together with the rarity of this condition hamper the clinical diagnosis. ${ }^{2}$ Here we present a case of splenic torsion in a 32 year young lady. Early recognition of the condition and timely surgical intervention are highlighted to prevent complications. ${ }^{5}$

\section{Case Report:}

A 32 years lady presented to the emergency department with pain and gradual distension of abdomen for 7 days. Pain was colicky in nature and was confined to upper abdomen. Abdominal pain and distension was aggravated even after taking liquids, and she had passed flatus several times after admission. She also had noticed a lump in her left upper abdomen for 7 days. She vomited several times, day before admission. She had no H/O haematemesis, melaena, fever and absolute constipation.

She had history of similar episodes of recurrent abdominal pain several times in the last six years and got herself admitted in different hospital and treated conservatively.

Abdominal examination shows slightly distended abdomen with visible lump and peristalsis. An ill-defined lump about $10 \mathrm{X} 8 \mathrm{~cm}$ felt in the left lumber region, which was firm, slightly tender , slightly mobile in side to side and dull on percussion. Bowel sound was sluggish. Her other systemic examinations revealed normal.

Investigation of abdominal radiograph shows distended bowel loops with no fluid levels.

Abdominal ultrasonography shows spleen was enlarged in size, absent at its normal position but located in the left lumber region and enlarged, suggestive of Ectopic spleen. Other organs are normal.

All other investigations within normal limits.

The patient underwent laparotomy, her spleen was found in ectopic position in left lumber region and there 


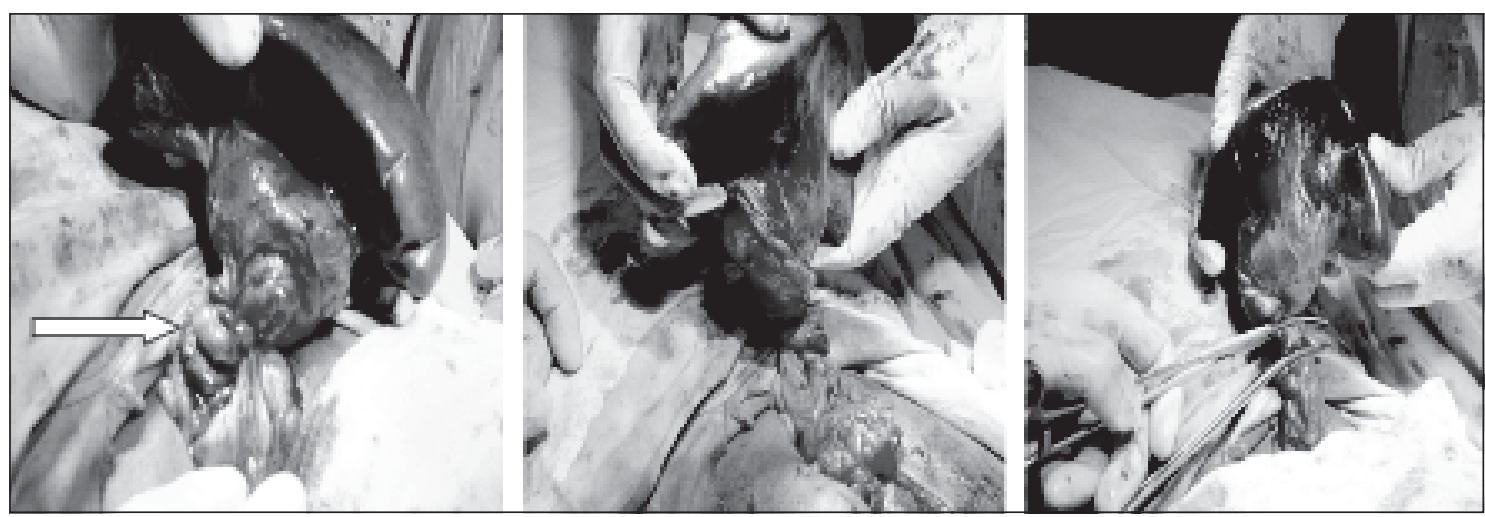

Fig.-1: (a) Torsion of a wandering spleen (b) Splenic detorsion done in anticlock wise direction. (c) Splenctomy done after clamping the splenic vascular pedicle.

was no attachment of spleen with stomach, left kidney and diaphragm. The spleen was floating and loops of small intestine adherent to the hilum of spleen and greater omentum was wrapped around the spleenic hilum resembling a lump. After releasing small intestinal and omental adhesion from spleen. There was 1 \& $1 / 2$ clockwise torsion of spleen around its vascular pedicle (Figure-I a). The twisted spleen was blackish in colour , signifying infarction. Detorsion of the splenic pedicle was done and hot mop was applied, but there was no change of splenic vascularity and colour. and splenectomy was done (Figure-I b, c)

The patient's recovery was uneventful .She was discharged on the 7 th POD with appropriate post splenectomy vaccination against pneumococcus and H.influenzae and long acting penicillins..

\section{Discussion:}

Wandering spleen is defined as mobile spleen that is attached only by an elongated vascular pedicle, allowing it to migrate to any part of the abdomen or pelvis. ${ }^{6}$

Synonyms - Ectopic Spleen, Displaced or Aberrent Spleen, Splenoptosis, Pelvic Spleen Free floating Spleen, Drifting Spleen. ${ }^{5}$

Wandering spleen is a rare clinical condition found in less than $0.5 \%$ of splenectomies that is characterized by ectopic positioning of the spleen within the abdomen or pelvis. $^{7}$

The first description of this entity is attributed to Van Horne, a Dutch physician in 1667 with confirmation by autopsy. ${ }^{8}$

Soleimani et al performed an extensive review of the literature from 1895 to 2005 finding 238 cases of wandering spleen, the majority of which were in the form of case reports and small case series. ${ }^{9}$

The incidence of wandering spleen is unknown and is difficult to determine. It usually occurs between the ages of 20 and 40 years and $70 \%$ to $80 \%$ of cases are seen in women; most are of reproductive age at the time. ${ }^{5}$

A male predominance has been found in children less than 10 years with $\mathrm{M}$ : $\mathrm{F}$ ratio of $6: 1$ while a female predominance is seen in adults. ${ }^{10,11}$

The spleen can 'wander' due to excessive mobility associated with ligamentous laxity of its peritoneal attachments. There are multiple causes of this ligamentous laxity including congenital and acquired etiologies. In the congenital form, there is failure of development of the normal splenic suspensory ligaments including the lienorenal and gastrosplenic ligaments. ${ }^{12}$ The acquired form can occur in conditions that weaken the ligaments including pregnancy with elevated estrogen levels and after trauma. ${ }^{13}$

Patients with a wandering spleen may be asymptomatic, present with a movable mass in the abdomen, or have chronic or intermittent abdominal pain because of partial torsion and spontaneous detorsion of the spleen. ${ }^{14,15}$

Torsion is the most common complication. Acute torsion usually presents as an acute abdominal pain, vomiting and fever. ${ }^{16}$ Clinically, the diagnosis can be suspected when a firm, movable abdominal mass is felt with the typically described "notched border". However splenic engorgement may hide the splenic notch. ${ }^{17,18}$

Preoperative diagnosis of wandering spleen is rarely suggested, based on clinical findings alone, because of 
nonspecific symptoms. Therefore, imaging plays a major role in establishing the diagnosis. ${ }^{15,18}$

Sonography showed the characteristic comma-shaped spleen in an ectopic position and the lack of splenic tissue in the left upper quadrant. Duplex Doppler color flow evaluation, provided optimal visualization of the organ and assessment of vascular supply. ${ }^{18}$

In CT scan whroled appearance of the splenic vessels and surrounding fat at the splenic hilum is considered as specific of torsion of a wandering spleen. ${ }^{19}$

Recently, magnetic resonance imaging and newer modalities such as liver-spleen scintigraphy, blood pool scintigraphy, and radio-labelled leucocytes have been tried. ${ }^{20,21,22}$

Definitive treatment for wandering spleen is operative, since non-operative treatment is associated with a complication rate as high as $65 \% .^{5}$ Sudden torsion may result in acute abdomen with life threatening complications like splenic infarction, gangrene, splenic abscess or rupture with a mortality rate as high as $50 \% .^{23}$ Other complications include gastrointestinal obstruction secondary to splenic adhesions or a long splenic pedicle, pancreatic necrosis, bleeding from gastric varices and abscess formation. ${ }^{13}$ Internal herniation of the wandering spleen has also been reported to cause recurrent abdominal pain. ${ }^{24}$

Initially, splenectomy was the treatment of choice. Currently, owing to overwhelming post-splenectomy sepsis and high mortality, splenectomy is indicated only if the blood supply to the spleen cannot be restored on detorsion. ${ }^{16}$ Splenopexy is a reasonable option when the spleen appears viable after detorsion and the splenic vein is not thrombosed. Results of splenopexy are considered good. ${ }^{25}$

Aiming to preserve splenic function, various techniques of splenopexy have been used. These include pexing the spleen by its capsule to the left upper quadrant laparoscopically, forming a post-erolateral extraperitoneal pocket at the level of 12th rib, mobilizing the splenic flexure of colon and then fixing the greater curvature of stomach to anterior abdominal wall and the use of polyglycolic mesh. ${ }^{24,26}$

Splenectomy should be done when there is no evidence of splenic blood flow after untwisting the splenic pedicle. ${ }^{27}$ In our case, spleen was totally black due to infarction and splenectomy was done.
Our patient's history together with the clinical and ultrasonological findings can serve as a reminder that the entity of wandering spleen should be suspected in unusual cases of recurrent unspecific abdominal pain. A high index of suspicion, prompt diagnostic workup and surgery are the key points for a favourable surgical outcome.

\section{Conclusion:}

The torsion of a wandering spleen is a rare abdominal emergency . Its diagnosis should be made in prompt time to prevent infarction of the spleen. Although torsion of a wandering spleen is an extremely rare clinical condition, it should be considered in the differential diagnosis of acute abdomen and one should intervene immediately to prevent splenic necrosis once diagnosis is made.

\section{References:}

1. Desai DC, Herba A, Davidoff AM, Schnaufer L, Wandering spleen : A challenging diagnosis. South Med J 1997 ;90: 439-43.

2. Hadas ltyel BmedSc BSc,Hananya Vaknine MD,Arie Judich MD,Mordechai Shimonov MD. Splenic Torsion of a Wandering Spleen. IMAJ 2011 ; 13 :119-120.

3. Sham Lal Singha,KN Rattan,Sachit Sharma,Satish Bansal.Torsion in a paediatric wandering spleen:Case report and review of literature.J Indian Assoc of Pediatr Surg 2007;12:30-1.

4. Maxwell -Armstrong CA, Clarke EDV,Tsang TM,Stewart RJ. The wandering spleen.Arch Dis Child 1996; 74:247-8.

5. Dahiya N,Karthikeyan D,Vijay S,Kumar T,Vaid M.Wandering spleen: Unusual presentation and course of events. Indian J Radiol Imaging 2002 ;12:359-62.

6. Hicham El Bouhaddouti, Jihane Lamrani, Abdellatif Louchi,Mounia El Yousfi,Noureddine Aqodad,Adil Ibrahimi,et al.Torsion of a Wandering Spleen. Saudi J Gastroenterol. 2010;16(4):288-291.

7. Sayeed S,Koniaris LG,Kovach SJ,HirokawaT. Torsion of a Wandering spleen.Surgery.Sep 2002; 132(3):535-536.

8. Soleimani M, Mehrabi A,Kashfi A, Fonouni H,Bucher MW,Kraus TW.Surgical treatment of patients with wandering spleen: report of six cases with a review of the literature.Surg Today.2007;37(3):261-269.

9. Lucas Dejohn,D.O. Hassan Hal,M.D,Louis Winner, M.D.Wandering Spleen: A Rare Diagnosis with variable presentation.Radiology case report USA 2008;3:229.

10. Sodhi KS,Saggar K, Sood BP,Sandhu P. Torsion of a wandering spleen : Acute abdominal presentation.J Emerg Med 2003;25: 133-7. 
11. Allen KB, Andrews G. Pediatric wandering spleen-the case forsplenopexy: Review of 35 reported cases in the literature.

12. Zahid Ahmad Hashmi, Dastageer Waheed, Sheikh Qais Falah. Torsion of a wandering spleen.Gomal journal of Medical Sciences Pakistan Jan-June 2010;8(1):93-94.

13. Robinson AP.Wandering Spleen : case report and review.Mt Sinai J Med. Oct 1998;55(5):428-434.

14. Ben Ely A, Zissin R, Copel L, Vasserman M,Hertz M,Gottlieb $\mathrm{P}$, et al. The wandering spleen : CT findings and possible pitfalls in diagnosis.Clin Radiol.2006;61:954-8.

15. Taori K, Ghonge N, Prakash A,Wandering spleen with torsion of vascular pedicle: early diagnosis with multiplaner reformation technique of multislice spiral CT. Abdom imaging.2004;29:497-81.

16. Stringel G, Soucy P, Mercer S. Torsion of the wandering spleen: splnectomy or splenopexy. J Pediatr Surg.1982;17: 373-5.

17. Matsumoto S, Mori H,Okino Y, Tomonari K, Yamada Y,Kiyosue H. Computed tomographic imaging of abdominal volvulus:pictorial essay. Can Assoc Radiol J. 2004;55: 297-303.

18. Fujiwara T, Takehara Y,Isoda H,Ichijo K,Tooyama N,Kodaira N,et al. Torsion of the wandering spleen: CT and angiographic appearance. J Comput Assist Tomogr.1995;19:84-6.

19. Raissaki M,Prassopoulos P, Daskalogiannaki M, Magkanas E, Gourtsoyiannis N. Acute abdomen due to torsion of wandering spleen: CT diagnosis.Eur Radiol. 1998;8: 1409-12.

20. Arda K, Kizilkanat K,Celik M,Turkalp E. Intermittent torsion of a wandering spleen in a child: The role of MRI in diagnosis.JBR-BTR 2004;87:70-2.

21. Shimizu M,Seto H,Kageyama M,Wu YW,Nagayoshi T,Kamisaki Y,et al.The value of combined 99mTc-Sn-colloid and 99m Tc-RBC scintigraphy in the evaluation of a wandering spleen. Ann Nucl Med 1995;9:145-7.

22. Posillico LF,Shah AN, A wandering spleen . Detection by in 111 leukocyte imaging. Clin Nucl Med 1996;21:287-9.

23. Moran JC, Shah U, Singer JA. Spontaneous rupture of a wandering spleen: Case report and literature review. Curr Surg 2003;60:310-2.

24. Andley M, Basu S, Chibber P, Ravi B, Kumar A.Internal herniation of wandering spleen- a rare cause of recurrent abdominal pain. Int Surg 2000;85:322-4.

25. Nemcek AA Jr, Miller FH, Fitzgerald SW: Acute torsion of a wandering spleen diagnosis by CT and duplex Doppler and color flow sonography. Am J Radiol. 1991;157;307-309.

26. Cohen MS, Soper NJ, Underwood RA, Quaseberth M, Brunt LM. Laparoscopic splenopexy for wandering(pelvic) spleen.Surg Laparosc Endosc 1998;8:286-90.

27. Alimoglu O, Sahin M, Akdag M. Torsion of a wandering spleen presenting with acute abdomen: a case report. Acta Chir Belg 2004;104:221-3. 